\title{
MASSIVE ATELECTASIS DUE TO FIBRINOUS BRONCHITIS BY
}

\author{
PAUL B. WOOLLEY \\ From Withington Hospital, Manchester
}

(RECEIVED FOR PUBLICATION MARCH 23, 1953)

Of all bronchial diseases, fibrinous, plastic, pseudo-membranous or Hoffmann's bronchitis seems to be one of the rarest. It has been estimated that up to 1869 about 130 cases had been reported (Walker, 1920); between 1869 and 1902 Bettmann (1902) collected 50, and between 1902 and 1920 a further 31 were reported (Walker, 1920). From 1920 to 1950 I have collected 25 new ones and these would bring the total up to about 136 in the world literature. The most important diagnostic feature is the coughing up of mucofibrinous casts during the course of a bronchitic type of illness. In some cases, however, casts are either not coughed up or not noticed ; as the acute variety may be fatal, they may be found in the bronchi at necropsy, as in the case described below.

Small fibrinous casts or plugs may be found in several acute or chronic lung diseases (Christian, 1919). This type of cast formation is symptomatic of the primary lesion and does not constitute true idiopathic fibrinous bronchitis. In the latter condition the casts are frequently long and may branch down to the seventh degree (Walker, 1920). They are greyish-white, sometimes hollow, and may contain Charcot-Leyden crystals and Curschmann's spirals, together with leucocytes and bacteria. The leucocytic infiltration is not uncommonly eosinophilic ; this has led to the belief that the condition may be allergic and closely associated with asthma (Beaumont, 1950); the bacteria are variable and of doubtful aetiological significance. A neurogenic origin has also been suggested and Bezancon has compared the disease with muco-membranous colitis.

In recent times it has been shown radiographically that extensive atelectasis may complicate the clinical picture. Rakower (1938) described a case with collapse of the whole of the left lung which subsequently expanded, and Malamud and Lisman (1946) described one with collapse of the left lower lobe. Both these patients coughed up casts and recovered. Johnstone (1945) described a fatal case in a girl of 21 years who had had no previous chest trouble; this illustrates the doubtful prognosis of the acute variety. The case reported below is another in which the diagnosis of fibrinous bronchitis was established after death.

\section{CASE Report}

On February 24, 1952, a retired woman school teacher, aged 61 years, was admitted to Withington Hospital because of cough of three weeks' duration, associated with considerable dyspnoea and rightsided chest pain. At the end of December, 1951, she had had what she described as a "bronchial cold" which had lasted two weeks but had cleared completely. However, three weeks before admission she developed what she thought was a further attack of bronchitis and stayed indoors. As she became progressively more dyspnoeic she called in her doctor, who diagnosed a consolidated right lower lobe and gave her $9 \mathrm{~g}$. of chloramphenicol with improvement. During these three weeks her temperature had been taken daily and was never above normal. Her sputum, initially yellow, had now become white and tenacious (about half a cupful daily). There had been no haemoptysis. Her weight had remained steady. She stated that she had a weak chest and had been subject to "bronchial colds" for a considerable time. She denied any previous attacks of pneumonia or asthma. There was nothing relevant in the family history.

On examination the temperature was normal and the respiratory rate slightly increased. There was a little rheumatoid arthritis of the fingers, but no clubbing. There was diminished expansion of the right side of the chest, with tracheal deviation to this side. The whole of the right lung was dull to percussion, and there was extensive bronchial breathing over the upper lobe. The heart sounds, which were normal, were just as loud to the right of the sternum as to the left; the blood pressure was 145/ $80 \mathrm{~mm}$. Hg. The abdomen and central nervous system were normal. The blood count showed: haemoglobin $81 \%$, leucocytes 8,000 per c.mm. Radiography of the chest showed complete opacity of the right hemithorax, with marked mediastinal shift to the right. A diagnosis of carcinoma of the bronchus causing total atelectasis of the right lung was made.

For three days after admission she remained afebrile and reasonably comfortable. The sputum was of a white, ropy consistency and not bloodstained. On the fourth day at 2 p.m. she suddenly had an attack of dyspnoea which was relieved by "cardophyllin" ; at 4.15 p.m. she had another, more severe, attack with considerable cyanosis. The dyspnoea was mainly inspiratory. The housephysician heard a flapping sound, coming and going with respiration, which made her think that there was something in the trachea causing the asphyxia. In spite of the administration of oxygen and analeptics the patient died shortly afterwards. 
NeCRoPSY.- The cardiovascular system showed no abnormality. The right pleural cavity contained about a pint $(560 \mathrm{ml}$.) of fluid, and the right lung was completely collapsed: there was some purulent bronchiolitis of the middle lobe. The left lung was congested. The whole of the right bronchial tree was occluded by a cast which branched down to at least the fifth degree, extended up to the tracheal bifurcation, grew over the latter and occluded the left main bronchus as well (Fig. 1). Although it

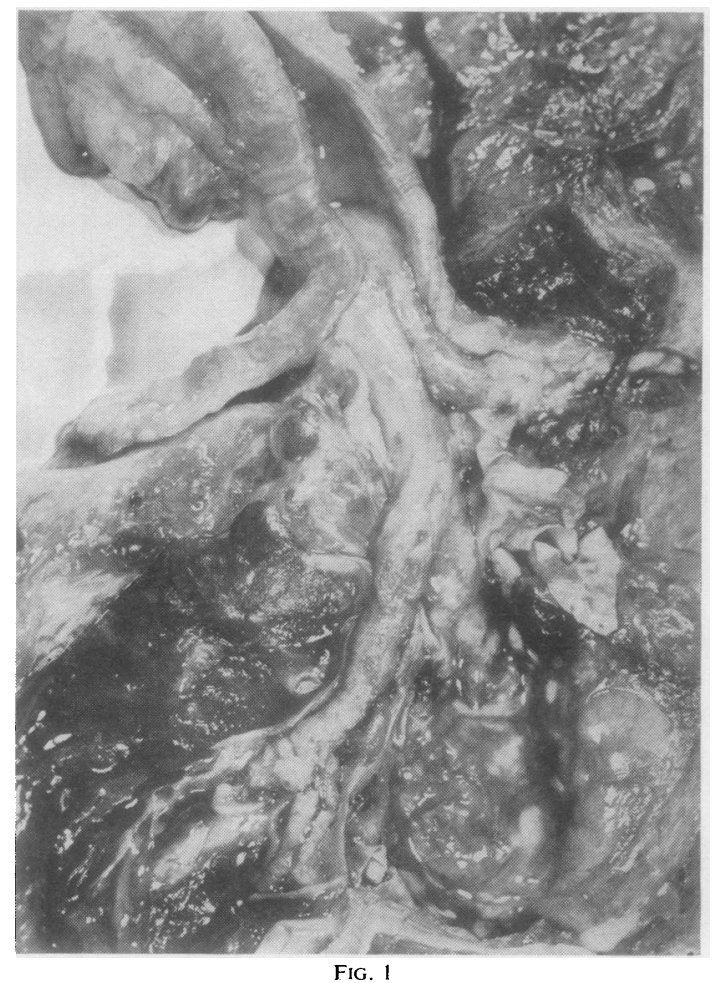

could easily be separated from the bronchial walls it was extremely tenacious; it was probably this property which prevented parts of it being expectorated and noticed. Cast formation on the left side was confined to some of the smaller bronchi. There were no other significant findings. Microscopical sections of the cast showed that it was muco-fibrinous and was infiltrated with leucocytes. Sections of the bronchial walls showed nothing abnormal.

\section{Discussion}

The total atelectasis indicated a blocked bronchus, and, as the patient had been apyrexial, the most likely diagnosis during life was bronchial carcinoma ; but at necropsy the block was found to be due to the large tree-like cast which finally asphyxiated her. The flapping sound heard during the second choking attack may well have been the "bruit de drapeau," due to vibration of a part of the cast in the air-stream. The absence of pyrexia in this case was noteworthy, and has been described in others.

The patient's statement that she had always been "weak-chested" might indicate that she had been subject to chronic fibrinous bronchitis, the last fatal episode being an exacerbation. Examination of the bronchial epithelium threw no light on the mode of formation of the cast. Cases have been described in which there was an excess of mucin cells, this excess being frequently associated with a damaged epithelium and outpouring of fibrin ; these two factors are regarded by several authors as the principal causes of cast formation.

The treatment consists of antibiotics, expectorants, and bronchoscopic aspiration. Frank (1937) has claimed a cure by the latter procedure and Jackson and Jackson (1934) have mentioned a case which required repeated bronchoscopic aspirations to relieve areas of collapse. The patient described above was to have been investigated bronchoscopically and she might well have benefited from aspiration once the diagnosis became obvious. Unfortunately, the main bronchi became occluded too quickly for this intervention to take place.

\section{SUMmary}

A case of fibrinous bronchitis is described in which total collapse of the right lung occurred.

The patient died from asphyxia due to occlusion of the carina before bronchoscopic aspiration could be undertaken.

I should like to thank Dr. J. S. Parkinson for advice and permission to publish, and Dr. L. Stent for pathological data.

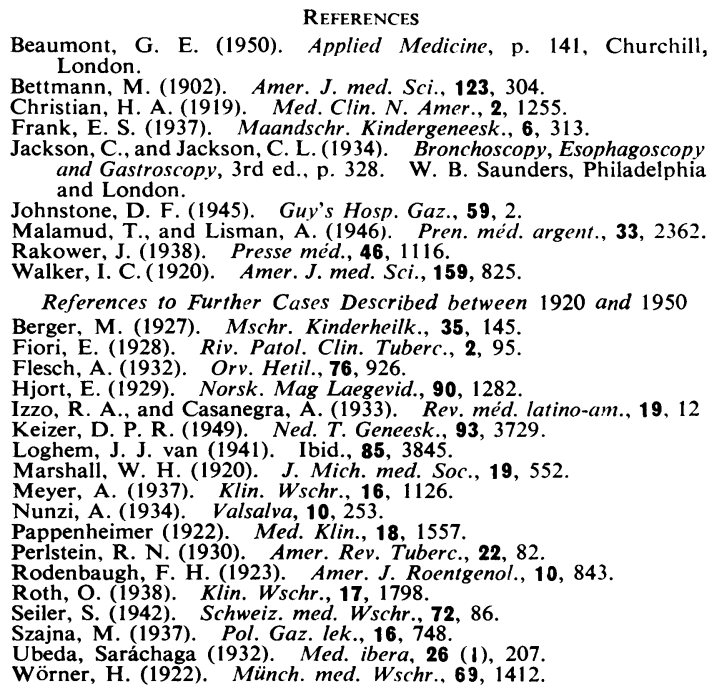

